\title{
SCIENTIFIC ARTICLES
}

\section{Microvascular reconstruction of oro-facial defects; a review of one surgeon's experience}

\author{
K. Ekanayake', M. Ekanayake ${ }^{2}$ \\ 1 University of Dublin, Ireland \\ 2 Teaching Hospital Peradeniya, Sri Lanka
}

Key words: Microvascular surgery; tracheostomy, reconstruction; free flaps; venous thrombosis

\begin{abstract}
\section{Introduction}

The practice of microvascular surgery is currently limited to a few centres in Sri Lanka. This type of surgery requires specialist training and a dedicated team. A retrospective review of the microvascular reconstructions carried out by one surgeon at the Dental Hospital, Peradeniya, Sri Lanka, was performed and the outcome has been analysed. The resection, neck dissections, the harvest of the flap and the reconstructions were performed single-handedly.
\end{abstract}

\section{Methods}

This is a retrospective study. Details were obtained from the theatre register, clinical notes and from the surgeon's personal data base. Patient identity, age, sex, diagnosis, type of surgery, reconstruction type, vein grafts, complications, anticoagulants used, type of anastomosis, tracheostomy, intubation period, flap salvage and final outcome were recorded.

\section{Results}

During the period from 2007 to 2010, 33 microvascular reconstructions were performed by the first author. Twenty two patients had resections for oral malignancies. The commonest flap was the fibula flap (16 cases), followed by the radial forearm flap (13 cases). There were four anterior lateral thigh flaps as well. The success rate was $82 \%$ with six failures. There were 21 males and 12 females. The average age was 44.7 , ranging from 14 years to 64 years. The male to female ratio was $2.7: 1$. Seven patients $(21 \%)$ were taken

Correspondence: K. Ekanayake

E-mail: ke@doctors.org.uk back to the theatre for exploration. Six patients developed thrombosis of the vessels. Only one flap thrombosis was identified in time and this was salvaged by re- anastomosis. No tracheostomies were performed electively. Patients were managed in the dedicated intensive care unit by keeping the endotracheal tube longer to secure the airway. Seventy five percent (75\%) of patients were extubated within 48 hours.

\section{Conclusions}

Microvascular surgery is technically highly demanding. The success rate $(82 \%)$ here is lower compared to more well-established units around the world. Three types of flaps have been used in this series. The commonest cause of flap loss was venous thrombosis. Proper training of nurses and junior doctors in recognising early signs of flap failure, close monitoring, prompt exploration when indicated and meticulous postoperative management during the first 72 hours would have given better flap survival. Tracheostomies can be avoided despite major head and neck resections. Ideally, two teams should work together to save time and minimise surgeon fatigue.

\section{Introduction}

Defects of the oro-facial region arise congenitally, from tumour resections or due to trauma. Reconstruction of these defects can be challenging due to the fact that the form and functions of the face and oral cavity have to be restored.

Tissue reconstruction can be performed with local flaps, pedicled flaps or with free flaps. Free flaps will require microvascular anastomosis. As such, it requires special training and a dedicated team.

In head and neck reconstruction, the commonly used free flap is the radial forearm free flap for soft tissue defects. However, anterior lateral thigh flap, rectus 
abdominus flap and latissimus dorsi flap are other alternatives. For hard tissue reconstructions, a fibula free flap has become the most popular flap. Composite radial forearm flap or deep circumflex iliac artery flap can also be used according to the size of the bony defect.

This review paper is a result of an audit conducted by the authors to identify the issues affecting microvascular surgery at the Dental Hospital, Peradeniya. All the resections and reconstructions were performed by the first author. The cases were performed between 2007 and 2010 during his tenure at this institution.

The author feels that it is important to review and publish this data as microvascular surgery is currently being performed only in a few centres in Sri Lanka. Microvascular free tissue transfer is highly demanding in terms of skill required of the surgeons as well as the supporting teams such as anaesthetists and nursing staff. In view of the complexity of the reconstructions undertaken and the stress of the length of surgery, surgeons need to regularly practise their surgical skills as the learning curve is quite steep.

\section{Radial Forearm flap (RFFF)}

The radial forearm flap was developed by the Chinese in the seventies. This flap has since gained wide popularity for soft tissue reconstruction of the oral cavity. It has a pliable thin skin and a long vascular pedicle base on the radial artery. One vena comitans gives adequate venous drainage. However, the cephalic vein is frequently used as well by some surgeons. A small part of the radius $(40 \%)$ can be incorporated into this flap as a composite graft for minor bony reconstructions. However, a radial fracture is a significant complication. This flap can be used as a sensate flap as well. A skin graft is required for the donor site and the two teams can work easily simultaneously.

\section{Anterior lateral thigh flap (ALT)}

This flap is based on the descending branch of the lateral femoral circumflex artery. Large defects can be reconstructed with this flap. It can be used as a fasciocutaneous or a myocutaneous flap. The donor site is well away from the surgical defect and therefore it is advantageous if two teams work simultaneously. Primary closure of the skin defect is possible in most cases.

\section{Rectus Abdominus flap (RA)}

This flap is based on the deep inferior epigastric artery from the external iliac system. As this flap incorporates the rectus muscle, a post-operative troublesome abdominal wall hernia is a possibility.

\section{Latissimus Dorsiflap (LD)}

A flap based on the thoracodorsal artery via the subscapular artery. This flap gives the best coverage for significant tissue losses. However, harvesting the flap requires positioning of the patient in a lateral decubitus position. As such, it is not possible to have two teams working simultaneously, adding to the surgical time.

\section{Fibula Free Flap (FFF)}

This is the most popular flap for the reconstruction of mandibular defects. This flap is based on the peroneal artery and venae commitantes accompanying it. The pedicle length can be extended according to the need. A skin paddle based on the lateral crural septum can be used to reconstruct the oral or extra oral defects. Donor site morbidity is very low, resulting in full function of the lower limb in most cases. This flap gives a significant length of bone for even full reconstruction of the mandible [1].

\section{Deep Circumflex Iliac Artery Flap (DCIA)}

Based on the deep circumflex iliac artery, this is an alternative to the fibula flap. The pedicle length can be short. The length will depend on the size and position of the bone flap and skin paddle. This flap gives plenty of bone bulk.

\section{Materials and methods}

A retrospective study was carried out on the patients treated by the first author. All patients were treated at the Dental Hospital, Peradeniya. The theatre register was used to identify the patients and the surgeon himself also maintained a database of all his cancer surgeries. Hospital clinical records were used to gather any further required information.

The following information was gathered:

Patient identity, age, sex, diagnosis, type of surgery, reconstruction type, vein grafts, complications, anticoagulants used, type of anastomosis, tracheostomy, intubation period, flap salvage and final outcome. 
Neck dissection was performed first, followed by resection of the lesion and the free flap was then harvested. The artery was irrigated immediately with heparinised saline. The anastomosis and reconstruction were performed last. Flaps were anastomosed within 4 hours of harvest using 9/0 ethilon or silk for the anastomosis. The author used a basic Lyca microscope for the anastomosis. The magnification varied from 2.5 to 5. End to end artery anastomosis and end to side anastomosis of the vein to the internal jugular vein was carried out as standard practice. The anastomosis site was irrigated with plain lignocaine and warm saline to prevent vasospasm. In every case the artery was anastomosed first. Every patient received a subcutaneous dose of Enoxaparin or Deltaparin just before the anastomosis and this was continued postoperatively for five days.

All patients were nursed in a dedicated intensive care unit. None of the patients had tracheostomies, but the airway was maintained post operatively by prolonged nasal intubation. The endotracheal tube remained in place until it was safe to extubate. Visual assessment was used daily as a guide to assess the patency of the airway. This was performed by inspecting the oropharynx. A joint decision was made by the surgeon and the anaesthetic team before extubating the patient.

Perfusion of the flaps was assessed by inspection of the colour and the consistency of the flap. Flaps were gently probed with an orange needle ( 25 gauge) and watched for bleeding. This was performed three times a day during the first 72 hours post operatively. Bright red blood appearing slowly was considered as an indication of good perfusion. Dark congested blood appearing rapidly is an early indication of venous congestion. Flap colour was not a good parameter to monitor as the majority of our patients had dark skin. However, flap congestion will lead to darkening of the skin paddle. This also causes a change in flap consistency to an oedematous look. Arterial problems will cause the flap to shrink and appear pale.

Capillary refill was only used to assess the flap in fair skinned patients. Although this is a great way to assess the perfusion, this cannot be used reliably in darker skinned patients. The temperature of the flap has no value in monitoring intra oral flaps as they are always bathed in saliva at body temperature.
The flaps were monitored three times a day by the same surgeon and needle probing was used as the gold standard. The ICU staff also assessed the flaps on an hourly basis. This was performed by inspection and with the use of a doppler. However, the doppler may give a false sense of security. An artery may be working well up to the anastomotic site and will give a strong positive sound, but there may be a clot at the site of anastomosis. Therefore, it is paramount that the doppler is used at a site distal to the anastomosis.

If there was any concern about the flap perfusion, nursing staff and the junior doctors were instructed to call the surgeon immediately. After further assessment, if indicated, the patient would be taken to the theatre for flap salvage by the author.

Intra- and post-operatively patients were given either Enoxaparin $40 \mathrm{mg}$ S.C or Deltaparin 5000 units S/C once daily. Adequate hydration was given and a good urine output was maintained. Unfortunately, we were not able to maintain a warm room temperature due to a faulty air conditioner. Haemoglobin $(\mathrm{Hb})$ was maintained around $10 \mathrm{~g} / \mathrm{dl}$ and transfusions were carried out only if $\mathrm{Hb}$ fell below 7-8g/dl.

Use of catecholamines was generally avoided. Hypotension was usually secondary to the blood/fluid loss or vasodilation and was treated with IV fluids.

\section{Results}

A total of 33 microvascular procedures were performed during a 7 year period, from 2003 to 2010 .

Twenty-two patients had excision of oral malignancies, neck dissections and reconstruction. Five patients had bilateral neck dissections. The majority of malignancies were squamous cell carcinomas with only one chondrosarcoma. There were also six ameloblastomas and one case of a recurrent keratocystic odontogenic tumour. Two patients had defects of the mandible due to trauma.

There were two children in this series. One was diagnosed with an aneurysmal bone cyst of the mandible and the other had an ossifying fibroma involving the mandible.

Sixteen flaps were fibula flaps, followed by thirteen radial forearm flaps and four anterior lateral thigh flaps 
as well.

There were 21 males and 12 females. The average age was 44.7 , ranging from 14 years to 64 years. The male to female ratio was $2.7: 1$. Seven patients $(21 \%)$ were taken to the theatre for re-exploration.

Post-operatively, ten patients were extubated within 24 hours. Fourteen patients were intubated for 48 hours. Four patients had the endotracheal tube in for 72 hours. Only three patients were intubated for more than three days. The longest intubation was for seven days in a patient who developed pneumonia. There were six failures resulting in a success rate of $82 \%$.

Results are summarised in Table 1 .

\section{Discussion}

There were 33 microvascular free flaps performed by the first author from 2007 to 2010 . Out of that 13 were radial forearm free flaps (RFFF). This flap was used mainly for the reconstruction of the tongue, floor of the mouth and the buccal regions. The radial artery was anastomosed to either the superior thyroid, lingual or to the facial artery. In all cases, the venous anastomosis was to one of the venae comitantes. The cephalic vein was not harvested routinely in order to cut down the time of harvest.

Sixteen flaps were fibula free flaps (FFF). The fibula flap with skin paddle was used for the reconstruction of the mandible.

The skin paddle based on the lateral crural septum was used to reconstruct the soft tissue defect resulting from the resection. In most cases this involved the floor of mouth. In one case the skin paddle was used to

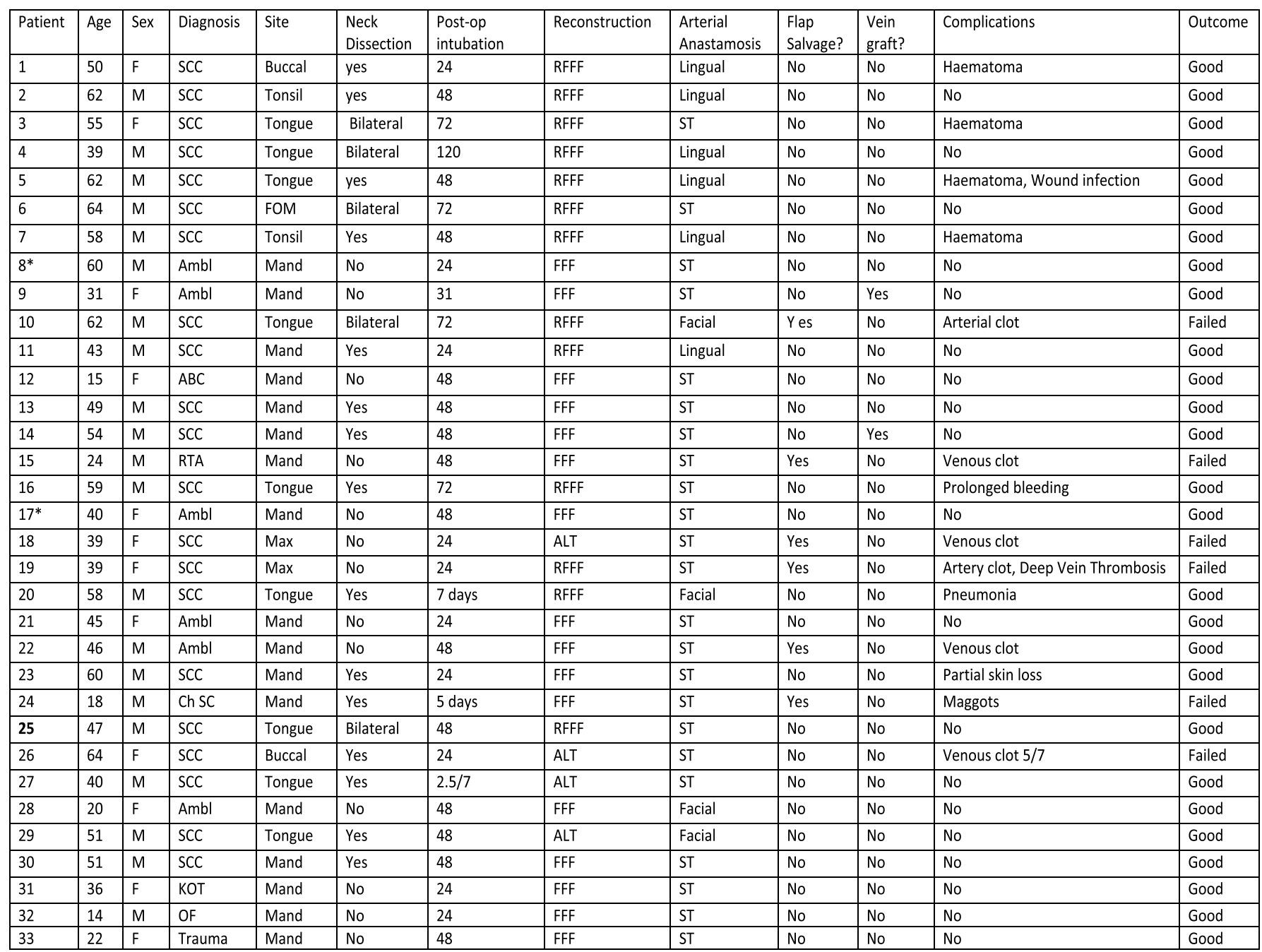

Table 1. Summary of results.

Key: SCC-Squamous Cell carcinoma, Mand-Mandible, Max-Maxilla, RFFF-Radial Forearm Free Flap, FFF- Fibula Free Flap, ALT-Anterio lateral Thigh Flap, ST-Superior Thyroid artery, Ambl-Ameloblastoma, OF- Ossifying Fibroma, ChScChondro sarcoma, ABC-Anerysmal Bone Cyst, RTA-Road Traffic Accident. 
reconstruct the buccal cheek excision. In a bilateral reconstruction of the mandible due to a war injury, the skin paddle was used temporarily to monitor the flap. After three months the excess skin in the submental region was excised [1]. Every fibula was harvested with an attached skin paddle to enable monitoring of perfusion.

There were four anterior lateral thigh (ALT) flaps. In three cases they were used to reconstruct the tongue and in one instance, it was used to reconstruct a maxillary/cheek defect.

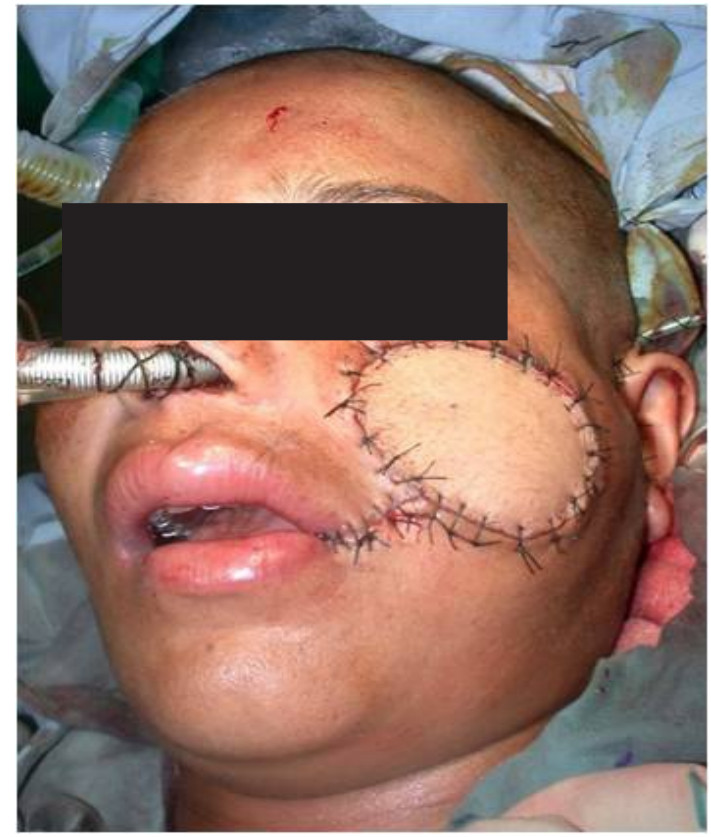

Figure 1. Reconstructed cheek/maxillary defect with ALT flap

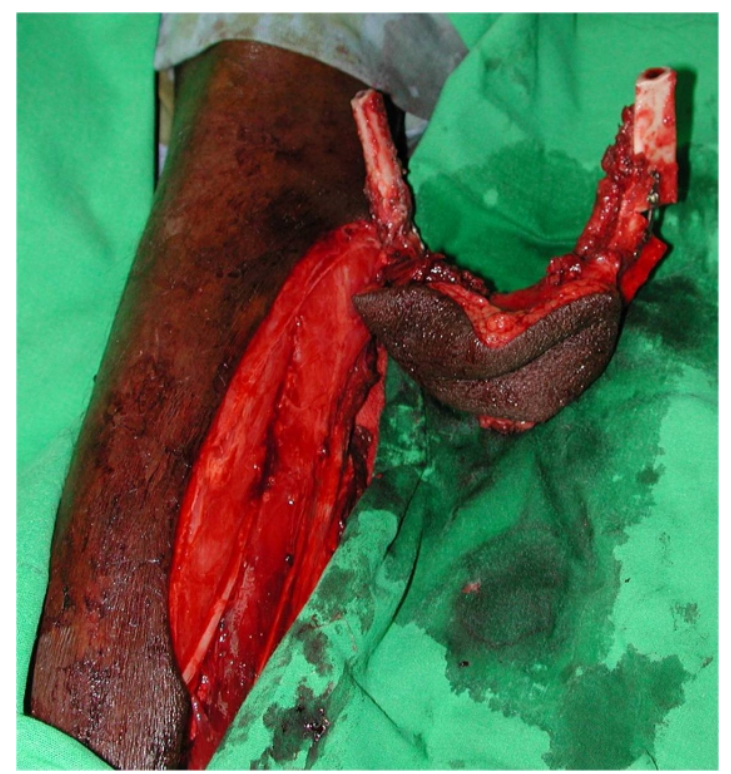

Figure 2. Fully contoured fibula to the shape of the mandible attached to the donor site
A vein graft had been used in only one instance for a fibula flap with a short pedicle.

The overall success rate was $82 \%$ with six failures. This is less than the standard success rate in major head and neck reconstructive units across the world. They have reported success rates of above $90 \%$. However, in a study conducted in Uganda (their first ever 19 consecutive cases) the success rate was 79\% [2]. This compares well with this study as the developing countries face similar constraints. It is clear that the success rate depends on the meticulous technique and the post-operative monitoring. One has to rely on the junior staff for early detection of the thrombosis and hence, staff training is vital. In most cases the culprit is a venous thrombus which occurs within the first 24 hours in most cases. There is little benefit in taking the patient back to the theatre when the clot is well established as the ischaemic damage has already taken place and the tissues will not survive. Re-establishment of the perfusion must take place within five hours to avoid the no-reflow phenomenon [3].

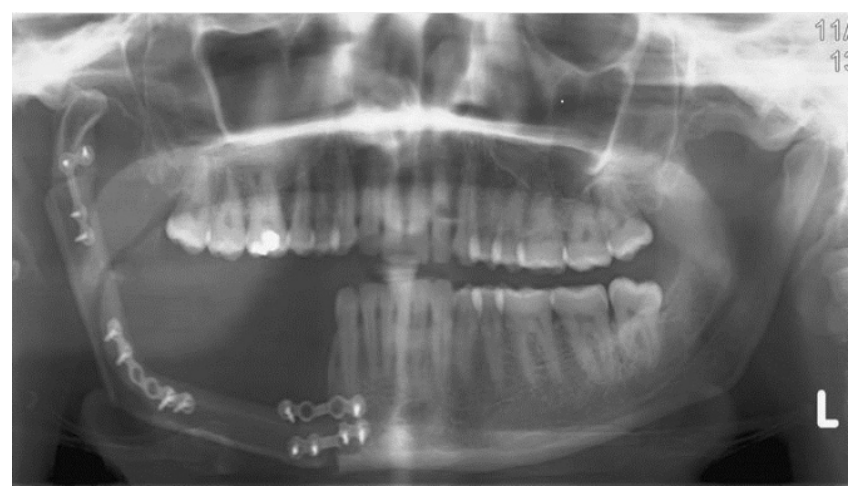

Figure 3. Radiograph of reconstructed right hemi-mandible

Studies state that the rate to return to theatre for flap salvage is $15 \%$ [4]. In this series, seven out of thirty three patients were taken back to theatre. This amounts to $21 \%$. Six patients developed thrombosis of the anastomotic vessels and the other was infested with maggots. Out of that only one flap was successfully salvaged. This flap regained perfusion after the vein was re-anastomosed. In the other five, there was a significant delay in recognising the complication which led to unsuccessful salvage. Had the thrombotic events been recognised in time it would have given a better outcome. This emphasises the need for close postoperative monitoring of free flaps. Monitoring should 
be done at hourly intervals during the first 72 hours by an experienced nurse. If there are any concerns, the consultant must be informed immediately. In our unit there was a learning curve and the flaps were monitored by inexperienced staff at the beginning. The author personally checked the flaps three times a day during the first 72 hours. In this series the majority of failures were due to venous clots. One flap failed on the 5th postoperative day. This is most unusual and the authors feel that dehydration was the cause as the patient was out of the ICU as the staff mistakenly felt that she was doing well. Therefore, proper hydration and careful fluid balance charting are vital, even after the first 72 hours.

All procedures, including resections, neck dissections, the harvest of the flap and the reconstruction, including anastomosis were performed by the first author. No trainee surgeons (registrars) were available to help. Therefore, it was lengthy surgery and surgeon fatigue was high.

It was not possible to regulate the temperature in the intensive care unit due to the faulty air conditioner and this was not favourable for flap survival as it was difficult to maintain a cold room temperature. This is not the optimal management of a post-operative patient with a free flap. The author recommends a warmer temperature during intra and post-operative periods.

One ALT flap failed (out of four) and the same patient suffered a failure of a radial forearm flap as well a week later. She later developed deep venous thrombosis of both lower limbs. It was believed that she was a thrombogenic individual and no further free flaps were considered for her. The defect was later closed with a cervico-facial flap.

Two fibula flaps failed out of 16 . One was following an attempt to reconstruct the whole mandible for a chondroscarcoma in an 18 year old man. The vessels were eaten away by maggots during the first 24 hours post operatively. The patient had received chemotherapy and radiotherapy prior to surgery and the fungating wound was infested by maggots at the time of surgery. Despite thorough excision and debridement a few maggots managed to hide under the flesh and appeared later to damage the flap.

Two radial forearm flaps failed out of 13 flaps. cases. There was a dedicated intensive care unit and it was possible to keep the endotracheal tube for an extended period of time. Twenty five (75\%) patients had the endotracheal tube extubated in 48 hours. One patient who developed respiratory difficulties had the tube in for seven days. However, if an ICU facility is an issue due to high patient demand, an elective tracheostomy should be performed [5].

The ideal criteria for post-operative management is:

- Haematocrit 30-35\%

- Normothermia

- High cardiac output

- Systolic blood pressure $(>100 \mathrm{~mm} \mathrm{Hg})$.

- Good urine output $>1 \mathrm{ml} \cdot \mathrm{kg}^{-1} \cdot \mathrm{h}^{-1}$

- $\mathrm{SpO} 2>94 \%\left(\mathrm{O}_{2}\right.$ in the first 24 hours $)$

- Hourly flap monitoring

- Adequate analgesia

\section{Conclusions}

This paper studied the outcome of microvascular reconstructions by a single surgeon in a Sri Lankan unit. This is the first published outcome of a series of microvascular reconstruction of head and neck defects in Sri Lanka.

The ability to reconstruct head and neck defects with free flaps is considered to be a necessary skill of all surgeons involved in head and neck resections. These surgeries are highly technique-sensitive procedures. Free flaps have many advantages, including the ability to get a wider clearance of the tumour without worrying about the extent of the resection. Ideally, two teams should work together to save time and minimise surgeon fatigue.

It appears that most oro-facial defects can be reconstructed with the help of three microvascular flaps, namely the radial forearm flap, the fibula flap and the anterior lateral thigh flap. Every fibula was harvested with an attached skin paddle to enable monitoring of flap perfusion.

There was no need for elective tracheostomy. If the facility is available however, prolonged post- operative intubation will help to avoid the complications of tracheostomies. 
Meticulous technique, proper instruments, close supervision of the flaps and optimal post-operative management of the patient will help in achieving a better success rate.

All authors disclose no conflict of interest. The study was conducted in accordance with the ethical standards of the relevant institutional or national ethics committee and the Helsinki Declaration of 1975, as revised in 2000.

No specific tests or personal information was included in the study. The information was analyzed from the surgeon's database.

\section{References}

1. Ekanayake K, Thomas D, Brennan P. Reconstruction of a Bilateral Mandibular Defect Due to Trauma Using a Single Fibula Free Flap. Journal of oral and maxillofacial surgery: official journal of the American Association of Oral and Maxillofacial Surgeons. 2013 ;71(10):1724-8.

2. Galiwango GW. Free Flap Surgery at Mengo Hospital, Uganda - A Review of The First 19 Consecutive Microvascular Free Tissue Transfers. East and Central African Journal of Surgery. Vol. 14, No. 1, March-April 2009, pp. 38-433.

3. Chang SY, Huang JJ, Tsao CK, Nguyen A, Mittakanti K, Lin CY, Cheng MH. Does ischemia time affect the outcome of free fibula flaps for head and neck reconstruction? A review of 116 cases. Plast Reconstr Surg. $2010 ; 126(6): 1988-95$.

4. Davison SP, Clemens MW, Kochuba AL. Anatomy of Free Flap Failures: Dissection of a Series. Modern Plastic Surgery, 2013: 3: 89-95.

5. Hammarfjord O, Ekanayake K, Norton J, Stassen LFA. Limited dissection and early primary closure of the tracheostomy stoma in head and neck oncology operations: a retrospective study of 158 cases. Int J Oral Maxillofac Surg. 2015; 44 (3) : 297-300. 